\title{
Correlates of Undeer Five Mortality in SNNPR Regional State, Spatial Data Analysis
}

\author{
Tizazu Toma Shamena, \\ Statistics Department, Wolaita Sodo University, P.O.Box:138, Sodo, Ethiopia
}

\begin{abstract}
The main aim of this study was to identify determinants of under-five child mortality, to describe spatial dependence of child mortality and develop models specifying risk factors used to diagnosis of child mortality among districts in SNNPR State of Ethiopia by using 2016 EDHS collected for 75 weredas/districts by employing spatial models. Spatial lag and spatial error model were fitted to the data, though spatial lag model specification was taken as the best fit for child mortality rate. Accordingly, from global and local spatial analysis it was found that children mortality rate in one district was directly affected by that of its neighbors. The results revealed that water closet, proportion of children under five, toilet availability, and mothers basic education attainment, vaccination coverage, size at birth, mother current working status, ORS information, altitude, stunting score and wasting score of children were significant determinants of children mortality rate. Thus, it is suggested that the geographically targeted preparation on accumulation of treatment that can be useful to control and stabilize spillover (nearest area spread) of disease such as diarrhea, malaria, and fever over space is recommended. It can be suggested for this states' that the government needs to make intervention to mitigate the spatial variation of the prevalence across regions.
\end{abstract}

Keywords: Child mortality, Spatial data Analysis, GEODA, EDHS, SNNPRS.

DOI: $10.7176 / \mathrm{JHMN} / 89-01$

Publication date:May $31^{\text {st }} 2021$

\section{Introduction}

\subsection{Back Ground of the Study}

Child mortality is a factor that can be associated with the well-being of a population and taken as one of the development indicators of health and socioeconomic status and also indicates a life quality of a given population, as measured by life expectancy as (Desta, M, 2011). Thus it is an area that many researchers focus and that has attracted the attention of policymakers and program implementer's worldwide. One of the most important targets of (MDGs) that introduced in 2000 at the United Nations Millennium Summit was reducing infant and underfive child mortality rates by two thirds from the 1990 levels by 2015. In 2000 the Ethiopian government announce the intention by signed the millennium declaration committing to achieve the (MDGs) by 2015, many of which overlap with the 2015 national policy goals, for instance, in 2000 the Ethiopian administer prepared child survival strategy and implementation plan to reduce under-five mortality of 140/1000 live births to 67/1000 live births by 2015 .

Most of the scholars indicated that the decline of mortality especially infant and child mortality in Ethiopia. According to Kenny and Kenny and Desta the mortality rate of children in Ethiopia has been declining. The main reason for this as they noted is the dawn ward of agriculture, the increase of urbanization which accelerates the economic performance of the country. Child mortality, also known as under-5 mortality, refers to the death of infants and children under the age of five. In 2010, 7.6 million children under five died, down from 8.1 million in 20098.8 million in 2008, and 12.4 million in 1990. About half of child deaths occur in Africa. Approximately 60 countries make up $94 \%$ of under-five child deaths as UNCEF, 2011. Death is often preceded by illness (morbidity). As a result, the state of health of individuals and societies is the prime determinant of mortality differences. However, variations in the types and About 472,000 Ethiopian children die each year before their fifth birthday, which places Ethiopia sixth among the countries of the world in terms of the absolute number of child deaths. Yet, there are effective low cost interventions to prevent two-thirds of these deaths (NSCSE, 2005). Children in the third world, especially in sub-Saharan Africa, usually suffer from more than one disease at a time. In most Countries of sub-Saharan Africa, the main causes of under-five deaths are more or less thesame. Ethiopia is a sub-Saharan Africa country with a land area of 1.14 million square kilometers. The size of the country and its location has accorded it with diverse topography, geographic and climatic zones and resources. With a projected population of 75.1 million in 2006, Ethiopia is the second most populous country in Sub-Saharan Africa (SSA). About $85 \%$ of the population resides in rural areas while the rest live in urban areas. According to the data of Millennium Development Goals Indicators Collected by the United Nations, IMR in 2007 at world level is 47; this rate is 5 for developed regions whereas 51 for the developing countries. Poverty is one of the most important factors affecting the under-five mortality rate in Africa. Ethiopia is one of the poorest African countries with, according to UNCEFreport, with a Gross National Income per capita of about \$220 in 2007. Infant and child mortality rates remain high, with most deaths being caused by easily preventable diseases, 
such as malaria, pneumonia and diarrhea.

\subsection{Statements of the Problem}

The study of under-five mortality becomes one of the most important researches in developing countries because there is high level of children mortality. There is much research on the patterns of determinants of under-five mortality, by analyzing how less than under-five mortality is differently affected by demographic, socioeconomic and environmental variables. This paper presents an analysis of the impact of demographic, socioeconomic and environmental variables on under-five mortality. The overall purpose of the paper is to determine the relative importance of various demographic, socioeconomic and environmental variables on under-five mortality in SNNPR. In this regard, the research questions of the interest were:

1. Does child mortality rate have spatial correlation?

2. Are socio-economic, demographic, environmental, and health related factors proximate characteristics related to experiencing of morbidity among children in the regions?

3. Do the effects of factors on mortality rate among children differ among districts?

\subsection{Objectives of the Study}

The main objective of this study is to identify determinants of child mortality, to describe spatial dependence of morality rate and develop spatial models specifying risk factors used to diagnosis of morbidity in SNNP Regional State.

\section{Specific objectives are:-}

- To determine important socio-economic and bio-demographic and environmental factors that contributes to morbidity including geographical effects as a surrogate for unobserved covariates with spatial information.

- To assess whether spatial association of mortality rate and its determinants exist in districts and also to identify territories of high risk.

- To provide scientific information to decision/policy based on empirical results

\section{Methodology}

\subsection{Study Area and population}

The Southern Nations, Nationalities and People's Region (SNNPR) is located in the Southern and south-western part of Ethiopia. It is bordered with Kenya in south, the Sudan in southwest, Gambella region in northwest and surrounded by Oromia region in northwest, north and east directions. The total area of the region estimated to be $110,931.9$ Square $\mathrm{Km}$ which is $10 \%$ of the country. Since 2007 the region total population is about $15,042,531$ accounting nearly $20 \%$ of the total population of the country; of population $49.7 \%$ are male and $50.3 \%$ are females (CSA, 2008). The population density of the region became 142 persons per sq.k.m, which makes the region one of the most densely populated parts of the country. The region is a multination, which consists of about 56 ethnic groups with their own distinct geographical location, language, cultures, and social identities living together. Among which Omotic and Cushetic are the most populous and diversified ones with the largest area coverage in region respectively. Based on ethnic and linguistic identities the region was divided into 16 zones sub-divided in to 126 weredas (districts), 22 administration town, and 8 special weredas with 3678 rural kebeles and 238 urban kebeles.

\subsection{Target Population}

The populations studied in this research were under-five children in SNNPR. A sample of 1277 children with their mother were used in 2016 Ethiopia demographic and health survey (EDHS, 2016).

\subsection{Variables Included in the Study}

In this study we employed two types of variables, these are dependent (response variable) and independent (Explanatory variables) variables.

Dependent variable

The dependent variable of child mortality (under-five mortality rate) is relative risk of dying in a specific age of below five years range of childhood)

\section{Independent variables}

Researcher used lot independent variables in study which was expected to cause child mortality in study area.

\subsection{Spatial Data Analysis}

In classical linear regression model $\mathrm{Y}=\mathrm{X} \beta+\mathcal{E}$, the response variable $\mathrm{Y}$ is assumed to be independent normal or Gaussian distributed and covariates, $\mathrm{X}_{1} \ldots \mathrm{X}_{\mathrm{P}}$ act linear on the response. By assumption the conditional 
expectation of $\mathrm{Y}$ is

$\mu=E\left(Y / X_{1}, X_{2} \ldots X_{p}\right)=\beta_{0}+x_{1} \beta_{1}+\ldots+x_{p} \beta_{p}$

Where $\mathrm{Y}$ is vector of dependent variable, $\mathrm{X}$ is designed matrix of predicators, $\beta$ is vector of parameters (coefficient)and $\varepsilon$ is vector of independently and identically distributed error terms.

$$
\mathrm{Y}=\left(\begin{array}{c}
\mathrm{y}_{1} \\
\mathrm{y}_{2} \\
\cdot \\
\cdot \\
\cdot \\
\mathrm{y}_{\mathrm{n}}
\end{array}\right), \quad \mathrm{X}=\left(\begin{array}{ccccccc}
1 & \mathrm{x}_{11} & \mathrm{x}_{12} & \cdot & \cdot & \cdot & \mathrm{x}_{1 \mathrm{k}} \\
1 & \mathrm{x}_{21} & \mathrm{x}_{22} & \cdot & \cdot & \cdot & \mathrm{x}_{2 \mathrm{k}} \\
\cdot & \cdot & \cdot & \cdot & & & \cdot \\
\cdot & \cdot & \cdot & & \cdot & & \cdot \\
\cdot & \cdot & \cdot & & & \cdot & \cdot \\
1 & \mathrm{x}_{\mathrm{n} 1} & \mathrm{x}_{\mathrm{n} 2} & \cdot & \cdot & \cdot & \mathrm{x}_{\mathrm{nk}}
\end{array}\right), \beta=\left(\begin{array}{c}
\beta_{\mathrm{o}} \\
\beta_{1} \\
\cdot \\
\cdot \\
\cdot \\
\beta_{\mathrm{k}}
\end{array}\right) \text {, and } \varepsilon=\left(\begin{array}{c}
\varepsilon_{1} \\
\varepsilon_{2} \\
\cdot \\
\cdot \\
\varepsilon_{\mathrm{n}}
\end{array}\right)
$$

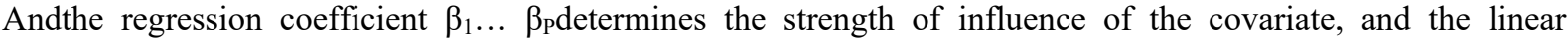
predictor $\mu$ is the sum of the covariate effect. Here each observation has an underlining mean of $\sum x_{i j} \beta_{i}$ and normally distributed random error term $\varepsilon$. Generally, the random error term $\varepsilon_{1} \varepsilon_{2} \ldots \varepsilon_{\mathrm{p}}$ has zero mean and uncorrelated variance covariance matrix $\varepsilon \sim \mathrm{N}\left(0, \delta^{2} \mathrm{I}\right)$ where $\delta^{2} \mathrm{I}=\operatorname{Var}(\mathrm{y})$ and an I is pxp identity matrix the assumption of independent observation also implies that $E\left(\varepsilon_{i} \varepsilon_{j}\right)=E\left(\varepsilon_{i}\right) E\left(\varepsilon_{j}\right)$.

\subsubsection{Quantification of Locations/Positions}

Contiguity information is quantified as contiguity (spatial neighbors) matrix that contains the elements 1 and 0 .

$$
\mathrm{C}=\left(\begin{array}{cccccc}
\mathrm{c}_{11} & \mathrm{c}_{12} & \cdot & \cdot & \cdot & \mathrm{c}_{1 \mathrm{n}} \\
\mathrm{c}_{21} & \mathrm{c}_{22} & \cdot & \cdot & \cdot & \mathrm{c}_{2 \mathrm{n}} \\
\cdot & \cdot & \cdot & & & \cdot \\
\cdot & \cdot & & \cdot & & \cdot \\
\cdot & \cdot & & & \cdot & \cdot \\
\mathrm{c}_{\mathrm{n} 1} & \mathrm{c}_{\mathrm{n} 2} & \cdot & \cdot & \cdot & \mathrm{c}_{\mathrm{nn}}
\end{array}\right)
$$

where: $\mathrm{n}$ is number of districts /locations under study $\mathrm{c}_{\mathrm{ij}}$ is element of $\mathrm{C}$ represents quantity local position of $\mathrm{i}^{\text {th }}$ and $\mathrm{j}^{\text {th }}$ districts for $\mathrm{i}, \mathrm{j}=1,2 \ldots \mathrm{n}$. Contiguity matrix $\mathrm{C}$ is constructed based on linear contiguity: define asc $_{\mathrm{ij}}=1$ for entities that share common edge to the immediate right or left of region of interest otherwise 0.

\subsubsection{Spatial Regression Model}

In the spatial linear regression model, spatial dependence can be incorporated in two distinct ways: as an additional regresor in the form of a spatially lagged dependent variable $\left(\mathrm{C}^{*} \mathrm{Y}\right)$ provide spatial lag model, or in the error structure $(\mathrm{C} \varepsilon)$ provides spatial error model. To test for spatial effects in models, spatial weights matrices are constructed and then included in a specified regression model. Thus, inference on the parameters allows one to explain the pattern for all locations as a function of exogenous variables (Cressie, 1993).

\subsubsection{Spatial Lag Model}

This is type of spatial regression which appropriate when the focus of interest is the assessment of the existence and strength of spatial interaction; suitable to filter out spatial dependence that comes from spatial spillovers. The matrix notation of the model is

$$
Y=\rho C * Y+\mathbf{X} \beta+\varepsilon
$$

where $\rho$ is a spatial autoregressive coefficient of the lag variable CY called spatial lag operator and given as $C Y=\sum_{j} C_{i j} Y_{j}$ where $\mathrm{C}_{\mathrm{ij}}$ is row standardized weight matrix corresponding to community pair $\mathrm{i}, \mathrm{j}$ hence $\sum_{j} C_{i j}=1, \forall i$. A random vector of error terms; $\varepsilon$ is independent and identically normally distributed with

mean zero and constant variance $\delta^{2} \mathrm{I}_{\mathrm{n}}$, for all I, Yis vector of dependent variables, $\mathrm{X}$ is a designed matrix of explanatory variables and $\beta$ is vector of coefficients of regression model. The single notation of the model is $Y_{i}=$ $\rho c_{i} Y+x_{i} \beta+\varepsilon_{i}$, where $c_{i}$ is the $i^{\text {th }}$ row of $C$. Parameters $\left(\beta, \delta^{2}\right)$ are estimated using maximum likelihood method of $\log$ likelihood function of transformed model and regressions was carried out along with a univariate parameter optimization of the concentrated likelihood function over values of the autoregressive parameter $\rho$. Given the above model as $Y=\rho C^{*} Y+X \beta+\varepsilon$, then the transformed model:A*Y $=X \beta+u$ implies $Y=A^{-1} X \beta+$ $\mathrm{A}^{-1} \mathrm{u}$ With $\varepsilon \sim \mathrm{N}\left(0, \delta^{2} \mathrm{I}\right), \mathrm{A}=(\mathrm{I}-\rho \mathrm{C})$ and $\mathrm{C}$ is row standardized spatial weights matrix. The log likelihood functions of transformed equation. 


$$
\begin{gathered}
\ln L(\beta, \rho, \sigma)=-\left(\frac{\mathrm{n}}{2}\right) \ln 2 \pi-\left(\frac{\mathrm{n}}{2}\right) \ln \sigma^{2}+\ln \|A\|-\frac{1}{2 \sigma^{2}}\left[(\mathrm{~A} * \mathrm{Y}-\mathrm{X} \beta)^{\prime}(\mathrm{A} * \mathrm{Y}-\mathrm{X} \beta)\right] \\
\text { Where, }\|A\| \quad \text { is determinant of matrix A, and } \mathrm{n} \text { is number of district under study. } \\
\operatorname{Or} L(\beta, \delta, \rho)=\ln |I-\rho C|-\frac{n}{2} \ln (2 \pi)-\frac{n}{2} \ln \left(\delta^{2}\right)-\frac{1}{2 \delta^{2}}\left(\left(Y-\rho C^{*} Y-X \beta\right)^{\prime}(Y-\rho C * Y-X \beta)\right)
\end{gathered}
$$

Maximizing the likelihood with respect to $\beta, \delta$, and $\rho$ gives the values of parameters that provide the highest likelihood of the joint occurrence of the sample dependent variable. Anselin (1988) suggest a way to maximize the log likelihood and get estimator for $\beta$ :

$$
\begin{aligned}
& \hat{\beta}_{s l}=\left(\mathrm{X}^{\prime} \mathrm{X}\right)^{-1} \mathrm{X}^{\prime} \mathrm{A}^{*} \mathrm{Y}=\left(\mathrm{X}^{\prime} \mathrm{X}\right)^{-1} \mathrm{X}^{\prime} \mathrm{X}^{*} \mathrm{Y}-\rho\left(\mathrm{X}^{\prime} \mathrm{X}\right)^{-1} \mathrm{X}^{\prime} \mathrm{C}^{*} \mathrm{Y} \\
& \hat{\beta}_{s l}=\hat{\beta} \text { ols }-\rho \hat{\beta}_{L}=\left(\mathrm{X}^{\prime} \mathrm{X}\right)^{-1} \mathrm{X}^{\prime}\left(\mathrm{Y}-\rho C^{*} Y\right)
\end{aligned}
$$

Where, $\hat{\beta}_{\text {ols }}=\left(\mathrm{X}^{\prime} \mathrm{X}\right)^{-1} \mathrm{X}^{\prime} \mathrm{X} * \mathrm{Y}$ is coefficientvector from OLS regression of $\mathrm{Y}$ on $\mathrm{X}$ with corresponding residual $\mathrm{e}_{\mathrm{ols}}=\mathrm{Y}-\mathrm{X} \hat{\beta_{o l s}}$, and $\hat{\beta}_{l}$-is the from OLS regression of $\mathrm{C}^{*} \mathrm{Y}=\mathrm{Y}^{\prime}$ on $\mathrm{X}$ and its corresponding residual $\mathbf{e}_{\mathrm{L}}=\mathrm{Y}^{\prime}-\mathrm{X} \hat{\beta}_{l}$. Maximum likelihood estimator of $\delta^{2}$ is $\mathrm{s}^{2}=\frac{1}{\mathrm{n}}\left(\mathrm{e}_{\mathrm{ols}}-\rho \mathrm{e}_{\mathrm{L}}\right)^{\prime}\left(\mathrm{e}_{\mathrm{o}}-\rho \mathrm{e}_{\mathrm{L}}\right)$ when $\rho$ is known.

We can now use all these to write down a version of the log-likelihood function in terms of $\rho$ only, the result is the Concentrated log-likelihood $(\operatorname{lnL} *)$

$$
\ln L^{*}=C O-\frac{\mathrm{n}}{2} \ln \left[\frac{1}{\mathrm{n}}\left(\mathrm{e}_{\mathrm{ols}}-\rho \mathrm{e}_{\mathrm{L}}\right)^{\prime}\left(\mathrm{e}_{\mathrm{ols}}-\rho \mathrm{e}_{\mathrm{L}}\right)\right]+\ln \|A\|
$$

CO doesn't involve unknown parameters and estimate of $\rho$ is obtained by maximizing $\ln L^{*}$ with respect to $\rho$. And also covariance-variance matrix of estimated parameters is estimated by maximum likelihood for large sample that attains Cramer's Rao lower bound. Moreover, clearly, we should be hesitant to make inferences about the effects of a covariate $\mathrm{x}_{\mathrm{i}}$ in a spatially lagged $\mathrm{Y}$ model without considering the spatial multiplier and the variation that will exist across spatial units (Ertur et al., 2007).

\section{Results and Discussion}

\subsection{Descriptive Results of Determinants}

Table1 below displays descriptive statistics for variables considered under study. The child mortality rate ranges from $0.00 \%$ (no mortality within 15 in that woreda) to $14.55 \%$ ( highly rate of mortality woreda/district) with mean 4.2 children died out of 100 live children and standard deviation of $5.59 \%$ in the Regional State. The drinking water closet in district varies from 2 minute to 996 minute with mean 123.81 minutes and standard deviation of 186.71 minutes. Percentage of mother who attended basic education ranges from $1 \%$ to $98 \%$ with mean $38.77 \%$ and standard deviation $26.63 \%$. Proportion of children ever vaccinated among district varies from $2.63 \%$ to $84.21 \%$ with mean $34.38 \%$ and standard deviation $16.36 \%$. Birth interval time from preceding and succeeding children ranges from 41.50 month to 88.00 month with mean birth interval 62.16 month and standard deviation 9.68 month. Table 1 also provided maximum, minimum, mean and standard deviation in percentage for factors: Proportion of household having protected toilet, Proportion of households disposing younger children stools when not using toilet, Proportion of children whose size at birth is below average, Proportion of children under five, Proportion of respondents heard about ORS, Percentage mother employed, Percentage mother currently working among districts. 
Table 1: Descriptive Statistics of Child Mortality Variation in Woreda/districts and Variables Considered Under Study (EDHS, 2016).

\begin{tabular}{|c|c|c|c|c|c|}
\hline Variables & $\mathrm{N}$ & Minim & Maxim & Mean & Std. Dev \\
\hline Mortality Rate & 75 & .00 & 14.55 & 4.20 & 5.59 \\
\hline Water closet in minutes & 75 & 1.92 & 996.29 & 123.81 & 186.71 \\
\hline Mother basic education attainment & 75 & 1.00 & 98.00 & 38.77 & 26.63 \\
\hline Proportion of Children vaccinated & 75 & 2.63 & 84.21 & 34.38 & 16.36 \\
\hline Proportion of hhd having toilet & 75 & 0.50 & 100.00 & 65.82 & 30.11 \\
\hline Proportion of hhd disposing stools & 75 & 5.88 & 100.00 & 65.242 & 22.01 \\
\hline Birth interval & 75 & 41.50 & 88.00 & 62.16 & 9.68 \\
\hline Proportion of respondent heard ORS & 75 & 0.50 & 99.50 & 59.72 & 24.85 \\
\hline Mother employed & 75 & 0.50 & 99.00 & 39.77 & 22.02 \\
\hline Wealth index factor score & 75 & -78405.00 & 255416.60 & -32043.70 & 895.95 \\
\hline Percentage of mother currently working & 75 & 2.00 & 90.60 & 32.77 & 31.02 \\
\hline Proportion of children below average size & 75 & 1.00 & 62.10 & 23.91 & 12.36 \\
\hline Proportion of children under five & 75 & 14.58 & 41.07 & 29.61 & 4.84 \\
\hline Number of hhd members & 75 & 3.73 & 7.89 & 5.94 & .78 \\
\hline Number of children under five & 75 & 1.50 & 2.67 & 1.75 & .33 \\
\hline Altitude & 75 & 979.00 & 3012.00 & & \\
\hline Stunting score & 75 & -2.28 & 3.70 & -1.13 & 0.57 \\
\hline Wasting score & 75 & -1.99 & 3.54 & -1.11 & 0.78 \\
\hline
\end{tabular}

From nutritional factor point of view stunting score and wasting score shows that stunting score ranges from -2.28 (severely stunted area) to 3.70 (normal) with overall mean -1.124 and variance 0.57 , and wasting score ranges from -1.99 (severely wasted area) to 3.5416(normal) with mean -1.11 and standard deviation 0.77 . Woreda's altitude also varies from 979 meter (low altitude) to 3012 meter (high altitude). And Mean wealth index factor score ranges from -78404.00 to 255217 with mean -32043.64 and standard deviation 58895.95 .

\subsection{Exploratory Spatial Data Analysis (ESDA) Results}

As presented in Table 2 the Moran's I scatter statistic for mortality shows those districts with above average mortality rates shares boundaries with neighboring districts that also have above average infection rates (HighHigh). It also shows those districts with below average mortality rates shares boundaries with neighboring districts that also have below average infection rates (Low-Low. And therefore, it leads us to observe that there is spatial autocorrelation in diarrheal rates in the regions.

Figure 1: Univariate Moron's Scatter Plot of Child Mortality Rates.

Table 2:Moron's Statistics of Child Mortality Rate.

\begin{tabular}{lccc}
\hline Variable & Moron's $\boldsymbol{I}$ & standardized value & P-value \\
\hline Mortality Rate & 0.5665 & 11.73211 & 0.001 \\
\hline
\end{tabular}

The theoretical mean of moron's stat is -0.072 and standard dev. 0.0489 for all variable obtained by $-1 / \mathrm{N}-1$

\subsection{Spatial Regression Analysis Results}

In this section we described a statistical model that incorporates spatial dependence raised from spatial lag explicitly by adding a spatially lagged dependent variable $\mathrm{Y}^{\prime}=\mathrm{CY}$ on the right hand side of the OLS regression equation $\mathrm{Y}=\mathrm{X} \beta+\varepsilon$ where $\mathrm{C}$ is spatial weight matrix. As a consequence, findings of this section allow us to understand which variable among the independent variables are related to the variation in mortality rates, and to explore the forms of these relationships.

Table 3 reported maximum likelihood estimate for determinants of diarrhea prevalence rate in spatial lag model, test statistic with corresponding p-value, and their standard errors that come from the heteroskedasticity consistent estimator of the covariance matrix of the maximum likelihood parameters. The significance of the coefficient of the spatially lagged dependent variable $(\rho)$ suggests that neighboring districts mortality rates are important determinants of a given district's mortality rate. This result is consistent with the findings of Frank (2006). More specifically, geographic spillover effects are important in our model of diarrhea morbidity rate variation.

Variables with positive and statistically significant effect on mortality rate are water closet (WC), proportion of children under five(PCU5), proportion children born below average size (PCBAV SIZE), where as variables with negative effect and statistically significant coefficients at $5 \%$ level of significance are proportion of children ever vaccinated(VACOV_100), proportion of household having controlled toilet(TOILET_100),proportion of households disposing younger disposal when not using toilet(DISPOSAL 
YOUNG_100), proportion of respondent heard about ORS(HEARD ORS),stunting score,wasting score and altitude.

Positive effect means that for a unit change in explanatory variable increase mortality rate in certain district by magnitude of estimate of parameter for that explanatory variable controlling for the effect of neighbor districts and other variable, whereas negative effect mean that for unit change in explanatory variable decrease mortality rate in district by magnitude of estimate of parameter for explanatory variable conditioning the neighbor districts mortality rate effect and other variable constant. For example for one minute increase in time to get drinking water (WC) in certain district increase mortality rate in that district by $0.00417 \%$ keeping other variable fixed. In another word, as the a distance to drinking water in a particular district increases by one minute, the possibility of mortality in that district increased by $0.00417 \%$. This result is similar with study conducted in Nekemte town, western Ethiopia, by Girma et al. (2008) and Chabala and Mamo(2001) that showed risk factors including distance of drinking water source (time taken to-and-from the source) appeared to be significantly associated with under-five childhood diarrheal morbidity.

The change in proportion of house hold having protected toilet and disposing young children stools were found to be negative and significant (at 5\% level). This result is similar with findings of Girma et al. (2008) and study done in SSA countries which showed that children in SSA living in households with some kind of toilet facility are less likely to experience diarrhea morbidity than children in households that do not have toilet facilities.

The parameter estimate 0.0542 for proportion of children under five(PCU5) indicates that a unit increase in proportion of children under five in district increase mortality in that district by $0.0542 \%$ keeping the effect of other independent variable constant. The sign tells that high proportion of children (below 5) in one district may aggravate population in the same area to participate in productivity discharge their responsibility to care them. These results share idea of (Berisha, 2011; Fahrrmeir and Khatab, 2007).

The coefficient estimate -0.0121 indicates that $1 \%$ increase proportion of household having protected toilet (TOILET_100) decreases mortality in distinct by $0.0121 \%$ remaining other variable influence constant.

The parameter estimate -0.005 for proportion of children ever vaccinated indicates that for $1 \%$ increase in proportion of children ever vaccinated in certain district decrease mortality rate in that particular district by $0.005 \%$ keeping others variables fixed. And Proportion of children born below average size (PCBAV SIZE) is significant and has positive effect on mortality rate of districts. This is due the fact that the greater the proportion of the children born below average size in district, higher the probability morbidity and mortality finally. Children who had low birth weight were more likely to be sick longer than infants who had appropriate birth weight or children born below average size can't defend childhood disease such as diarrhea, fever and malaria. This result is consistent with study done Teshager (2011) using 2005 EDHS and similar with the study done in Northeast Brazil by Lira et al. (1996) who showed that children with low birth size experienced 33\% more days with diarrhea and $32 \%$ more days with vomiting.

In basic knowledge point of view proportion of mothers who attained basic education (EDAT_100), proportion of respondents who heard about ORS (HEARD_ ORS) both have negative and significant effect among district. Nevertheless percentage mother educated primary and above determine mortality rate in certain district, some mothers have no basic education skills which of course necessarily need education for mother. The same interpretation was given for ORS information.

The negative coefficient and significant value for stunting score and wasting score indicates that the less stunt score or waste score the higher mortality rate. Most literature shows that stunting and wasting are problem that highly correlated with childhood morbidity. A vicious cycle between mortality and stunting means that children with stunted were more likely to attacked by diarrhea .The results may be due to shortage of nutrition leads to non health (more vulnerable to diarrhea).

For altitude interpretation is slightly different because it is fixed for certain district and is fixed effect to diarrhea prevalence in certain district; therefore coefficient can be interpreted as districts with high altitude are less likely to be infected by diarrhea. This is may be due to polluted water flow from high to low (see discussion part for more details).

Table 3 also present measures of fit for models in discussion part. R-square $=0.5682$ which tells us that $56.82 \%$ of variation in diarrhea prevalence rate was explained due to variation in the explanatory variable in the model and spatial lag dependent variable.

Finally Table 3 contains Diagnostics Tests Spatial lag Model of Mortality Rate. Three measures that are included to maintain comparability with the fit of spatial lag models that are the log likelihood (-60.83), the Akaike information criteria(147.653) and Schwarz criteria(186.261). These three measures are based on assumption of multivariate normality and corresponding likelihood function for standard regression model. The higher log likelihood, the better the fit (less negative). For the AIC and SC information criteria the direction is opposite, the smaller the measure, the better the fit. 
Table 3: Maximum Likelihood Estimate for Factors of Mortality Rate in Spatial Lag Model (EDHS, 2016).

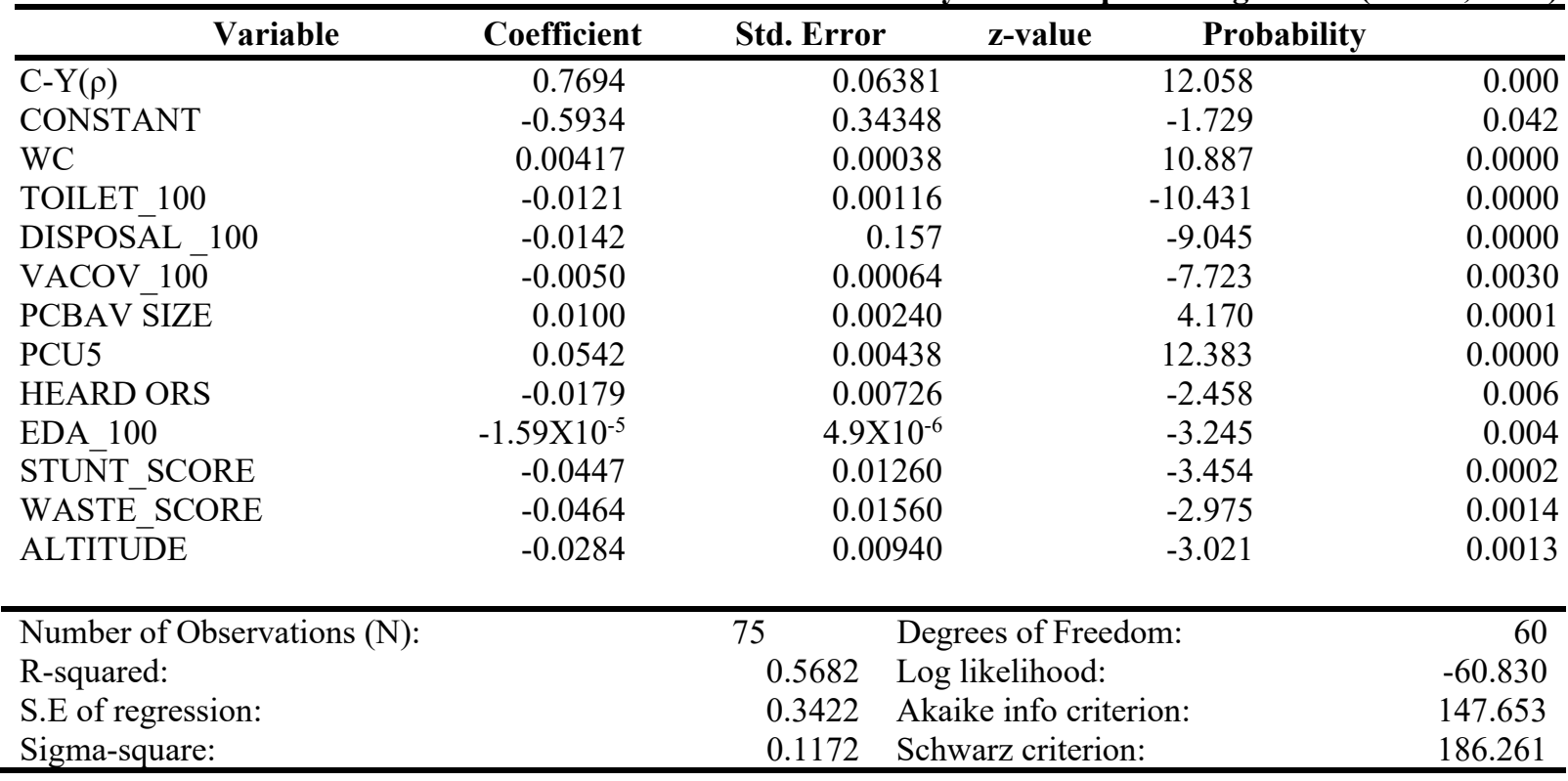

\section{CONCLUSIONS AND RECOMMENDATIONS}

\subsection{Conclusions}

Geographically close districts with similar socio-economic and demographic characteristics and vulnerability dimensions are more conducive to grouping forces, such as using of unprotected drink water. The clustering of underlying disease dimensions might be due to a number of reasons including sanitation that has been applied to groups of areas or socio-economic issues that lead to spatial clustering of mortality rate.

Our estimation results of spatial lag model for mortality rate indicate that water closet, proportion of children under five, protected toilet availability, mothers basic education attainment, vaccination coverage, size at birth, mother current working, ORS information, altitude from sea level, stunting score and wasting score of children have significant influence in explanation of mortality rate differentials across districts in the regions.

\subsection{Recommendations}

Based on findings and other related literatures study we forward the following recommendations to mitigate regional mortality.

$>$ The expanded program on immunization should be spread out to reduce child mortality in study area.

$>$ The study suggests that efforts should be made to build public and private toilet facilities.

$>$ Improvements in access to clean water and adequate sanitation, along with the promotion of good hygiene practices (particularly hand washing with soap) can help to prevent childhood diarrhea morbidity and mortality in turn.

$>$ The study also suggest that basic education to mother, improve the nutritional status of children, community participation on hygiene and sanitation will be important measure in geographically targeted preparation to reduce possibilities of occurrence morbidity.

$>$ Further study will recommend to be conducted by incorporating time or employing other forms of spatial models and including other excluded districts.

Thus, we suggest that the most effective policy mix for alleviating differences amongst the regions districts mortality rate, balancing hospital composition and providing sanitation, and encouraging community to participate in sanitation and environment protection. Furthermore reducing average family, encouraging mother to take basic education in regions may reduce morbidity of child and mortality in turn.

\section{References}

1. Desta, M., 2011. Infant and child mortality the role of Socioeconomic, Demographic and Biological factors In the previous five years period of 2000 and 2005.

2. Desta, M., 2011. Infant and child mortality the role of Socioeconomic, Demographic and Biological factors In the previous five years period of 2000 and 2005.

3. Ethiopia Demographic and Health Survey, 2005. Central Statistical Agency, Addis Ababa, Ethiopia, RC Macro, Calverton, Maryland, USA, September 2006.

4. Ezra, M. and Gurum, E., 2002. Breastfeeding, birth intervals and child survival: analysis of the 
1997community and family survey data in southern Ethiopia.

5. Goro, M., 2007. The stalling child mortality: the case of three northern regions. The 5th conference of union for Africa population, Tanzania.

6. Hala, A.-A., 2002. Water and health in egypt: An empirical analysis. Phd thesis.

7. "UNCEF, 2011.Unicef annual report 2011.Hill, K., Bicego, J., and Mahy, M., 2001. Childhood mortality in Kenya: An examination of trends and determinants in the late 1980s to mid1990s.

8. Hosmer, D. W. J. and Lemeshow, S., 1989. Applied logistic regression. New York, USA: John Wiley \&Sons. p. 307.

9. Schultz, T., 1984."Studying the impact of household economic and community variables on child mortality."Population and Development Review, vol. 10, pp. 215-35.

10. International Baby Food Action Network Africa, 1999. IBFAN Africa statement on HIV and infant feeding.The IBFAN Africa regional workshop on policy guidelines for infant feeding and HIV: 23-27.

11. Kenny, A. and Kenny, C., 2006. "Life, liberty and the pursuit of utility: Happiness in Philosophical and economic Thought London: Imprint Academic.

12. Kombo, J. and Ginneken, V., 2009. Determinants of infant and child mortality in Zimbabwe: Result of multivariate hazard analysis.

13. Kumar, P. and Gemechis, 2010. "Infant and child mortality in Ethiopia: As statistical analysis approach."

14. Manda, S. O. M., 1999. "Unobserved family and community effects on infant mortality in Malawi."Genus, vol. LIV pp. 143-164.

15. Millennium Development Goals Report. Challenges and prospects for Ethiopia.Ministry of Finance and Economic Development (MOFED) of the Federal Democrat Republic of Ethiopia and the United Nat ions Country Team, March 2004. Addis Ababa

16. Mosley, W. and Chen, L., 1984. "An analytical framework for the study of child survival in developing countries."Population and Development Review, vol. 10, pp. 25-45.

17. Mturi, A. J. and Curtis, L. S., 1995. "The determinants of infant and child mortality in Tanzania."Healthpolicy plan, vol. 10, pp. 384-94.

18. Mutunga, C. J., 2004. Environmental determinants of child mortality in Kenya.Kenya Institute for Public Policy Research and Analysis (KIPPRA), Nairobi, Kenya. 18.Ridder, G. and Tunali, I., 1999. "Stratified partial likelihood estimation."Journal of Econometrics, vol. 92, pp. 193-232.

19. Sahn, D. E. and Stifel, D. C., 2003. "Exploring alternative measures of welfare in the absence of expenditure data."Review of income and wealth, vol. 49, pp. 463-489.

20. Schultz, T., 1984. "Studying the impact of household economic and community variables on child mortality."Population and Development Review, vol. 10, pp. 215-35.

21. Baah, 1994.A study of infant, child and maternal mortality in Ghana.Ghana statistical service incollaboration with Ministry of Health and UNICEF, Accra, Ghana.pp. 24-32.

22. Twum - Baah, 1994. A study of infant, child and maternal mortality in Ghana.Ghana statistical service incollaboration with Ministry of Health and UNICEF, Accra, Ghana.pp.24-32.

23. UNCEF, 2009. The state of the world's children: Maternal and newborn health. 23. "UNCEF, 2011. Unicef annual report 2011.

24. World Bank, 2007. Capturing the demographic bonus in Ethiopia: Gender, Development and Demographic Actions, Washington, DC. 